\title{
RESEARCH HIGHLIGHT Developments in pluripotency: a new formative state
}

\author{
Stefan H. A. Hoogland ${ }^{1}$ and Hendrik Marks ${ }^{1}{ }^{1}$ \\ Cell Research (2021) 31:493-494; https://doi.org/10.1038/s41422-021-00494-w
}

\begin{abstract}
During development, mouse embryonic cells progress in around 4 days from a naïve to a primed pluripotent state. A recent study in Cell Research by Wang and colleagues reports the in vitro capture of an intermediate pluripotent state, represented by so-called formative pluripotent stem cells, revealing the dynamic nature of the progression of pluripotency.
\end{abstract}

Pluripotency refers to the cellular potential to differentiate into any of the three germ layers, ectoderm, endoderm or mesoderm, which together give rise to a mature organism. However, pluripotency is not restricted to a single cellular phase, but consists of a dynamic continuum during which cells mature and become responsive to specification cues. ${ }^{1,2}$ Until recently, pluripotency was categorized into two distinct phases: "naïve" and "primed". ${ }^{1,2}$ Naïve pluripotency is established in the inner cell mass of the pre-implantation embryo, with in vitro representatives known as embryonic stem cells (ESCs). Following implantation, naïve pluripotent cells polarize ${ }^{3}$ and undergo morphological, metabolic and epigenetic changes to mature into a primed pluripotent state. ${ }^{4}$ This state is established in the postimplantation epiblast, represented in vitro by epiblast-derived stem cells (EpiSCs). ${ }^{5}$ Although ESCs and EpiSCs are functionally pluripotent and can form teratomas, EpiSCs show reduced contribution to chimeras and germline competence is lost. ${ }^{5}$ Epigenetically, the transition from naïve to primed pluripotency is accompanied by a wave of DNA methylation, and by random $X$ inactivation occurring in female cells. In addition, the transition is accompanied by alterations in signaling and a metabolic switch from bivalent glycolytic and mitochondrial respiration in naïve pluripotency to exclusively glycolytic respiration in primed pluripotency. ${ }^{4}$ In mice, these changes happen within a relatively short time frame of $\sim 4$ days, between embryonic day (E) E3.5 and E7.5. However, what happens within this time frame? In 2017, the existence of an intermediate form of pluripotency, coined formative pluripotency, was hypothesized. ${ }^{1}$ On one hand, the further maturation towards formative pluripotency would prepare naïve ESCs for lineage differentiation. On the other hand, formative pluripotent cells were supposed to be able to differentiate towards primordial germ cells (PGCs; the early precursors of gametes), a potential that is lost in primed EpiSCs. ${ }^{1}$ In a recent paper published in Cell Research, Wang et al. ${ }^{6}$ now report the establishment of in vitro mouse formative pluripotent stem cells (fPSCs), generated from either E5.5-6.5 epiblasts, ESCs (Fig. 1) or induced pluripotent stem cells (iPSCs).

To enable capturing the formative pluripotent state, Wang et al. ${ }^{6}$ use the growth factors Activin A and Fgf2, similar to what is used for mouse EpiSCs. ${ }^{5}$ However, the powerful use of Matrigelbased three-dimensional (3D) culture conditions, combined with inhibition of the Wnt/ $\beta$-catenin pathway, allows to stably maintain formative epiblast-like embryoids, referred to as fPSCs, which show polarization and lumen formation. The fPSCs are functionally pluripotent in that they contribute to chimeras, are able to form teratomas and are capable of in vitro differentiation towards either of the three germ layers. Importantly, the fPSCs as established by Wang et al. ${ }^{6}$ can efficiently differentiate into PGCs, a unique feature as compared to ESCs and EpiSCs. Epigenetic characterization showed that the fPSCs are close to primed EpiSCs: the fPSCs contain high levels of DNA methylation and elevated levels of $\mathrm{H} 3 \mathrm{~K} 27 \mathrm{me} 3$ at bivalent promoters of genes that are poised for activity, ${ }^{7}$ while female fPSCs contain an inactivated $X$ chromosome. However, fPSCs expressed high levels of formative pluripotency markers, such as Otx2 and Fgf5, and low levels of primed pluripotency and lineage-specific markers including Foxa2, Brachyury/T and Sox1. Naïve pluripotency makers such as Tfcp2l1, KIf2 and Esrrb are expressed at low levels in the fPSCs. Further global transcriptome comparisons revealed that the fPSCs are most similar to E5.5-E6.5 epiblasts. Altogether, the authors convincingly show that they were able to capture an in vitro pluripotent state intermediate of "naïve" and "primed", resembling a formative pluripotent state.

In 2020, three other groups have reported the pioneering of 2D culture conditions which support stable in vitro derivation and maintenance of intermediate pluripotent embryonic states. ${ }^{3,8,9}$ All these states show cell polarity, a transcriptome indicative of formative pluripotency and, as far as assayed, ${ }^{8}$ glycolytic metabolism. Surprisingly, comparative analysis of the transcriptomic and epigenomic data generated by these groups ${ }^{3,8,9}$ and in the current study ${ }^{6}$ show that the intermediate pluripotent ESCs in each of the culture conditions resemble different time points in the naïve-to-primed transition, over a timespan ranging from approximately E5.0 to E6.5 ${ }^{6}$ (Fig. 1). General trends within this timespan include a gradual increase in DNA methylation levels and a gradual switch from naïve transcription factor (TF) expression towards formative TF expression, seemingly followed by a more immediate switch towards lineage-biased TF expression in primed EpiSCs. ${ }^{3,6,8,9}$ Interestingly, based on discriminative features, the pluripotent states as reported in these studies could be categorized into a more naïve-like intermediate state and a more primed-like intermediate state, the latter including the fPSCs (Fig. 1). In contrast to the naïve-like group, the primed-like intermediate cells (i) show a developmentally more advanced transcriptome; (ii) are not capable of re-converting to naïve pluripotency (as far as assayed); (iii) do not show germline transmission in vivo; and (iv) show $X$ inactivation in female cells. ${ }^{3,6,8,9}$ Altogether, these in vitro cell systems provide an excellent model to comprehensively characterize the dynamic molecular processes associated with developmental progression of embryonic cells throughout pluripotency, and to understand how lineage commitment is executed.

${ }^{1}$ Faculty of Science, Department of Molecular Biology, Radboud Institute for Molecular Life Sciences (RIMLS), Radboud University, Nijmegen 6525GA, The Netherlands
Correspondence: Hendrik Marks (h.marks@ncmls.ru.nl)

Published online: 17 March 2021 


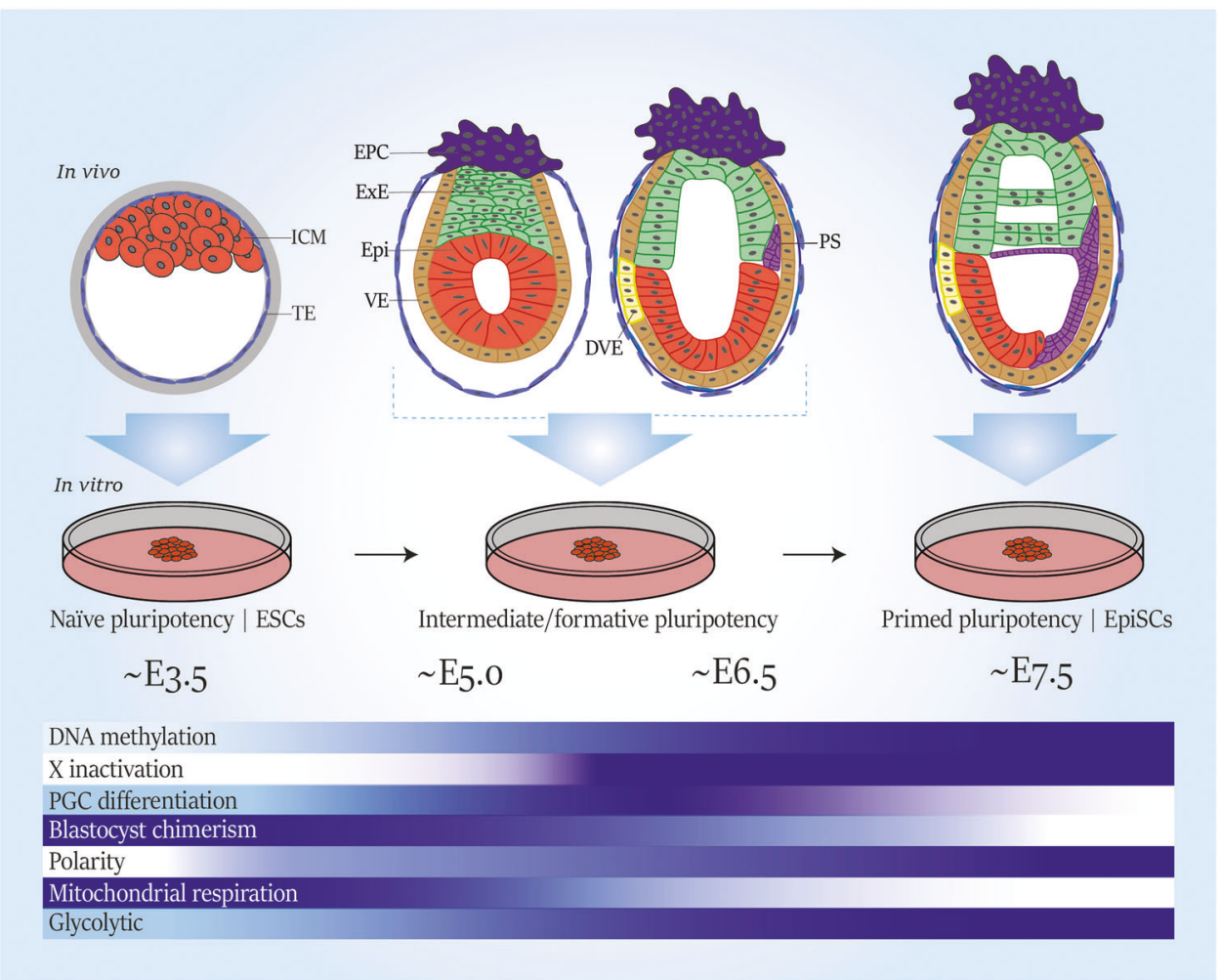

Fig. 1 The three defined pluripotent states. Formative pluripotency represents an intermediate embryonic pluripotent state, reflecting mouse E5.0-E6.5, with features distinct from naïve and primed pluripotency. ICM inner cell mass, TE trophectoderm, EPC ectoplacental cone, ExE extraembryonic ectoderm, Epi epiblast, VE visceral endoderm, DVE distal visceral endoderm, PS primitive streak.

The establishment of mouse formative ESCs provide a unique opportunity to further understand early post-implantation development. An important question is whether the results as obtained for mouse formative pluripotency can be extrapolated to other species, in particular to human. While it remains to be tested whether the culture conditions as pioneered by Wang et al. ${ }^{6}$ support derivation and/or maintenance of fPSCs in human, two recent studies have reported human intermediate-like pluripotent stem cells using other cell culture conditions. ${ }^{8,9}$ However, due to challenges of functionally testing human pluripotent cells and of staging these by the use of in vivo embryonic epigenomic and transcriptomic features, it remains difficult to properly benchmark human pluripotent cells. But with their better potential to respond to directed differentiation as compared to other human pluripotent ESCs, the establishment of human fPSCs might have important implications for regenerative medicine.

\section{REFERENCES}

1. Smith, A. Development 144, 365-373 (2017).

2. Hackett, J. A. \& Surani, M. A. Cell Stem Cell 15, 416-430 (2014).

3. Neagu, A. et al. Nat. Cell Biol. 22, 534-545 (2020).

4. Weinberger, L., Ayyash, M., Novershtern, N. \& Hanna, J. H. Nat. Rev. Mol. Cell Biol. 17, 155-169 (2016)

5. Brons, I. G. M. et al. Nature 448, 191-195 (2007).

6. Wang, X. et al. Cell Res. https://doi.org/10.1038/s41422-021-00477-x (2021).

7. van Mierlo, G. et al. Cell Stem Cell 24, 123-137 (2019).

8. Yu, L. et al. Cell Stem Cell https://doi.org/10.1016/j.stem.2020.11.003 (2020).

9. Kinoshita, M. et al. Cell Stem Cell https://doi.org/10.1016/j.stem.2020.11.005 (2020). 